\title{
Perivascular Epithelioid Cell Tumor of the Retroperitoneum in a Young Woman Resulting in an Abdominal Chyloma
}

\author{
T. E. Lans • G. H. van Ramshorst • J. J. Hermans • \\ M. A. den Bakker • T. C. K. Tran • G. Kazemier
}

Received: 10 December 2007 / Accepted: 13 December 2007 /Published online: 23 January 2008

(C) 2007 The Author(s)

\begin{abstract}
Perivascular epithelioid cell tumor (PEComa) is an extremely rare neoplasm which appears to have predominancy for young, frequently Asian, women. The neoplasm is composed chiefly of HMB-45-positive epithelioid cells with clear to granular cytoplasm and usually showing a perivascular distribution. These tumors have been reported in various organs under a variety of designations. Malignant PEComas exist but are very rare. The difficulty in determining optimal therapy, owing to the sparse literature available, led us to present this case. We report a retroperitoneal PEComa discovered during emergency surgery for abdominal pain in a 28 -year-old Asian woman. The postoperative period was complicated by chylous ascites that was initially controlled by a wait-and-see policy with total parenteral nutrition. However, the chyle production gradually increased to more than 41 per day. The development of a bacterial peritonitis resulted in cessation of production of abdominal fluid permitting normal nutrition without chylous leakage. Effective treatment for this rare complication of PEComa is not yet known; therefore, we have chosen to engage in long-term clinical follow-up.
\end{abstract}

\section{Keywords PEComa $\cdot$ Chylous $\cdot$ Retroperitoneum}

\section{Introduction}

Perivascular epithelioid cell tumors (PEComas) are extremely rare mesenchymal neoplasms. They are characterized by immunoreactivity for both smooth muscle and melanocytic markers, such as smooth muscle actin, HMB-

T. E. Lans $(\bowtie) \cdot$ G. H. van Ramshorst • T. C. K. Tran •

G. Kazemier

Department of Surgery, Erasmus MC-University Medical Center, 'sGravendijkwal 230,

3015 GD Rotterdam, The Netherlands

e-mail: T.Lans@ErasmusMC.NL

\section{J. J. Hermans}

Department of Radiology,

ErasmusMC-University Medical Center,

Rotterdam, The Netherlands

M. A. den Bakker

Department of Pathology,

ErasmusMC-University Medical Center,

Rotterdam, The Netherlands
45, and melan-A. Histologically, PEComas are composed of cells with copious clear-to-eosinophilic cytoplasm that frequently shows perinuclear condensation. ${ }^{1}$ PEComas are a family of related mesenchymal neoplasms that including angiomyolipoma, lymphangioleiomatosis, clear cell "sugar" tumor of the lung, and another group of rare, morphologically and immunophenotypically similar lesions arising at a various visceral and soft tissue sites. ${ }^{2}$ Over the past decade, perivascular epithelioid cells (PECs) and tumors composed of these have engendered significant discussions and controversies with respect to their very existence as a clinico-pathological entity, their histogenesis, pathogenesis, and nomenclature. It appears now that these tumors may potentially arise in any anatomic location, as PECs do not have a normal anatomic homologue. Despite considerable advances in the recognition of these unusual lesions, the origin of these tumors remains elusive, and additional cases with longer clinical follow-up must be evaluated before their behavior can be predicted accurately.

In this case study, we describe a patient with a PEComa presenting with localized intra-abdominal chylous pockets probably caused by obstruction of lymphatic vessels. This combination and presentation has not been described before. 


\section{Clinical History and Histological Findings}

A 28-year-old Korean woman presented with left lower abdominal quadrant pain that had been present for 1 day. Her previous medical history was unremarkable, and laboratory investigations showed no abnormalities except for a high $\mathrm{C}$-reactive protein in the absence of fever. She initially underwent a laparoscopy in a community hospital after a transvaginal ultrasound revealed a mass in the lower left abdominal quadrant, which was interpreted as a strangulated left ovary.

Intraoperatively, a rubbery gray mass measuring $15 \mathrm{~cm}$ in diameter was found. This mass was located in the retroperitoneum, posterior to the left broad ligament. During biopsy, murky white fluid was released. The left ovary, uterus, and other intra-abdominal organs appeared normal. Postoperatively, she developed pain in the left groin. A computer tomography (CT) scan revealed an encapsulated fluid collection in the left lower abdominal quadrant, which was considered to be an abscess (Fig. 1). This collection was drained under ultrasonographic guidance. During the following days, progressive leakage of white fatty fluid emerged through the drain. This white fluid was diagnosed as chyle.

She was placed on a high-protein and low-fat diet containing exclusively medium-chain triglycerides. As she did not respond to the above measures, she was referred to our hospital. Soon after her admission, the drain became obstructed, after which, she developed acute abdominal pain and a high fever. An emergency laparotomy was performed. Remains of the earlier described mass were identified, and an attempt was made to remove the lesion completely. The previous operation and the ongoing inflammation prohibited complete removal, and again, a drain was placed. Postoperatively, there was persistent chylous leakage. Attempts to manage this by total parenteral nutrition and fasting were again unsuccessful.

The biopsy taken at the initial laparoscopy revealed nonspecific chronic inflammation. The surgical specimen taken at the laparotomy was routinely processed for histology. This biopsy contained tumor predominantly composed of spindle-shaped cells arranged in fascicles. These spindled cells, with eosinophilic cytoplasm, resembled smooth muscle cells (Fig. 2). There was mild pleomorphism, and focal necrosis was noted. Mitoses were sparse (two per $2 \mathrm{~mm}^{2}$ ). Lymph node metastases were not found. Immunohistochemically, the cells strongly expressed Vimentin, Desmin, smooth muscle actin, BCL-2, and HMB-45 and were negative for cytokeratin, S-100, and Melan-A. A diagnosis of PEComa was established.

Extensive examination revealed neither a different primary site of the tumor nor metastatic lesions. Despite optimal conservative measures, chylous leakage persisted, progressing to more than 21 per day.

A second laparotomy was performed aimed at controlling the chylous discharge. During this procedure, the origin of the chylous leakage was identified after provocation with an extensive amount of double cream through the nasogastric tube. Macroscopically, no tumor was present, and only extensive chylous leakage was noted from the retroperitoneum (Fig. 3). An attempt was made to occlude all lymphatic vessels from the left groin to the diaphragm
Figure 1 CT-scan abdomen with chyloma. Drain in situ in lower left abdomen.

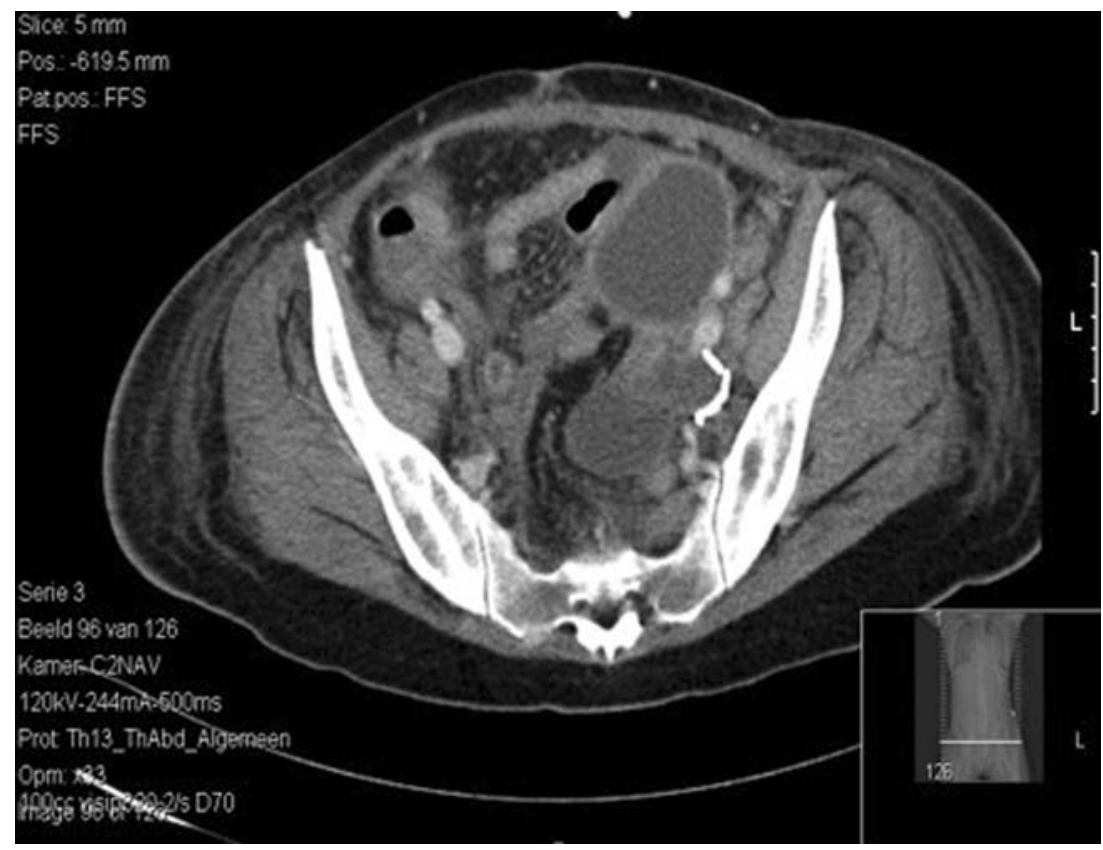


Figure 2 Histology. Photomicrograph of HE stained slide showing plump spindle cells with fibrillar eosinophilic cytoplasm with focal vacuolation. The nuclei are regular with small nucleoli. Insets: left, desmin immunoperoxidase stain showing intense generalized staining; right, HMB45 immunoperoxidase stain showing focal cytoplasmic reactivity.

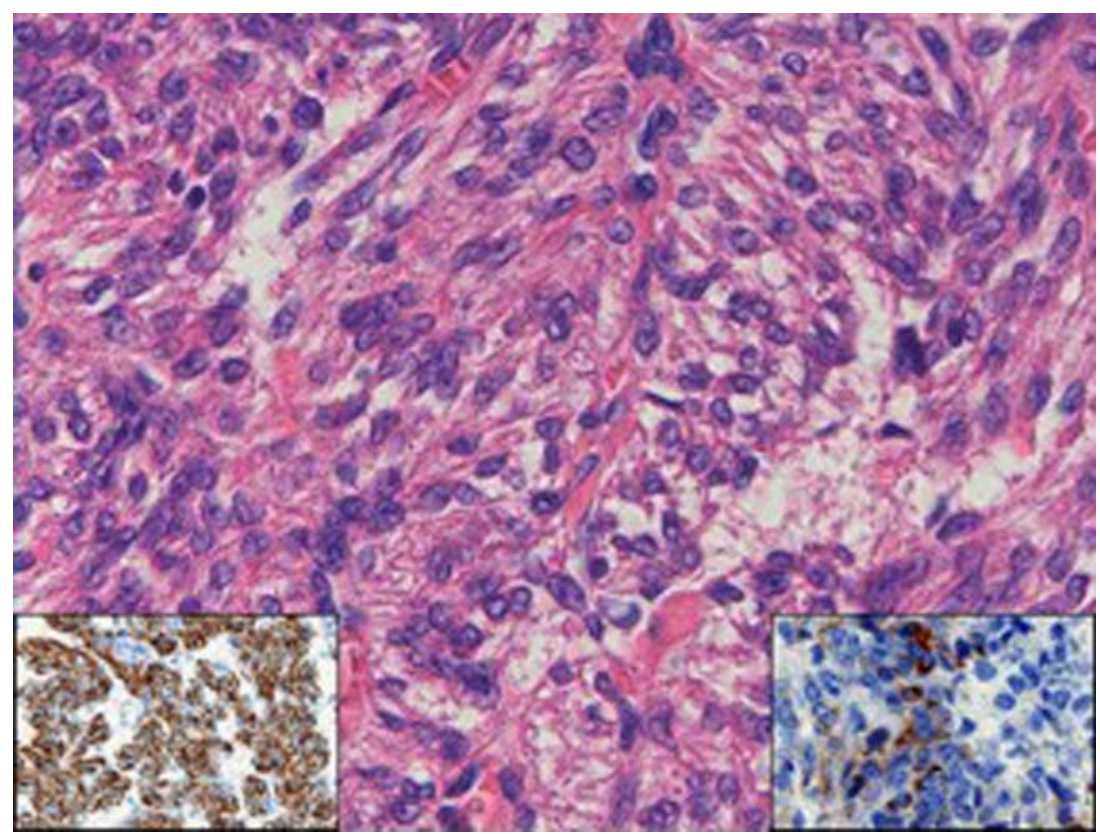

with sutures and titanium clips, as the leakage extended proximally after every clip. Finally, no leakage was noted; therefore, no drain was placed in this procedure.

Postoperatively, she developed peritonitis and septicaemia within $24 \mathrm{~h}$, necessitating re-operation. At relaparotomy, there was still chylous leakage but no additional pathology. A drain was placed which produced more than 11 of chyle per $24 \mathrm{~h}$. She developed another episode of peritonitis and sepsis after the drain became blocked, and further surgery was not considered effective. The drain was removed. She was treated with intravenous antibiotics (Vancomycin and Tazobactam). After 3 weeks, the chylous leakage had finally ceased. Her diet was carefully brought back to normal, and she was discharged from the hospital 5 months after admittance. On the CT scan, there was no evidence of tumor recurrence, and there is still no evidence of tumor 1 year postoperatively. Annual CT scans will not be performed regarding the young age of this patient.

\section{Discussion}

As relatively few malignant PEComas have been reported, firm criteria for malignancy have yet to be established.
Figure 3 Intraoperative findings (head is positioned on left side). Chyloma can be seen as white structures behind the uterus.

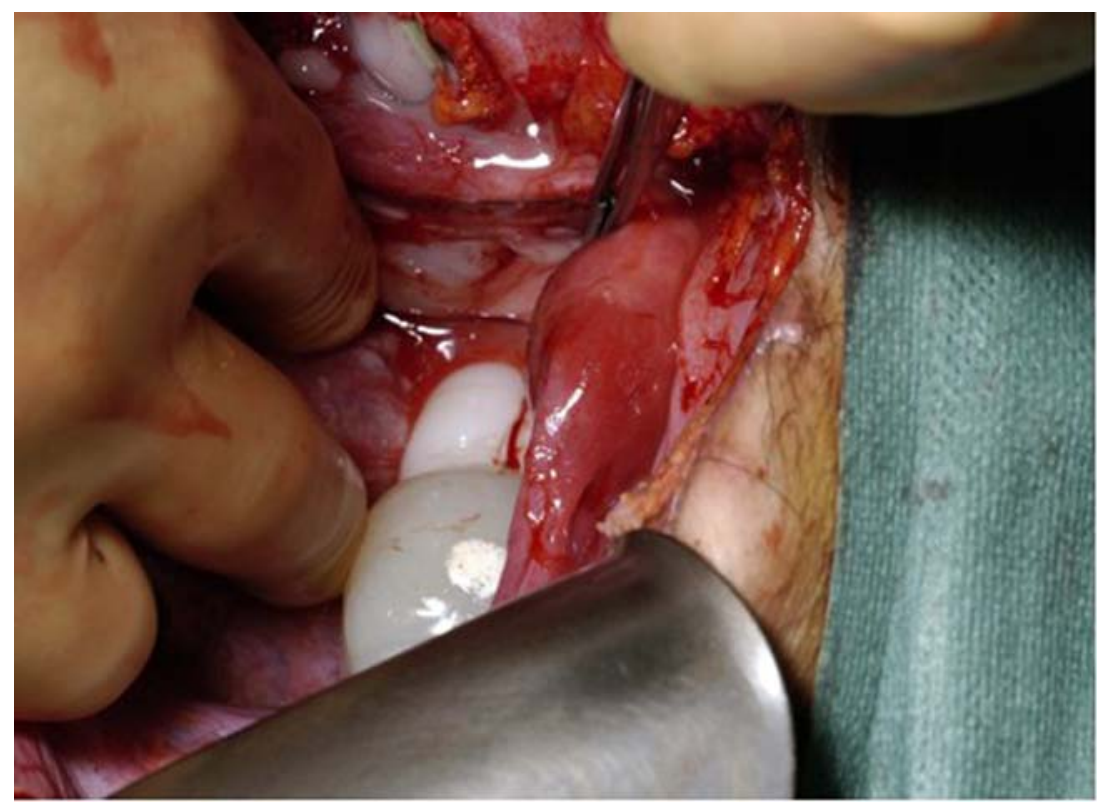


However, in a recent study published in abstract form (presented at the United States and Canadian Academy of Pathology conference in 2005), Folpe et al. ${ }^{3}$ evaluated criteria for malignancy in 24 PEComas arising at a variety of visceral and somatic soft tissue sites. The authors found that local recurrence and/or metastasis were associated with large tumor size, more than $70 \mathrm{~mm}$ in diameter, necrosis, and a mitotic index exceeding 1 per 50 high-power fields. Infiltrative growth and marked hypercellularity are other features that have been reported to be suggestive of malignancy in other cases. In addition, marked pleomorphism and nuclear atypia may indicate malignant behavior. Thus far, PEComas are considered tumors of uncertain malignant potential. ${ }^{4}$ Spread of this tumor to other sites might become evident even several years after primary manifestation. ${ }^{5}$

Chylous ascites is a rare form of ascites resulting from an accumulation of lymph in the abdominal cavity. It is caused by an interruption in the lymphatic system. The diagnosis is established when the concentration of triglycerides in the ascitic fluid exceeds $200 \mathrm{mg} / \mathrm{dl} .{ }^{6}$ Chylous ascites may occur due to different mechanisms, including (a) obstruction of the lymph flow caused by external pressure (i.e., compression by a mass) causing leakage from dilated subserosal lymphatics into the peritoneal cavity; (b) exudation of lymph through the walls of dilated retroperitoneal vessels lacking valves, which leak fluid through a fistula into the peritoneal cavity as in congenital lymphangiectasia; or (c) traumatic thoracic duct damage causing direct leakage of chyle through a lymphoidperitoneal fistula. An underlying malignancy is a common cause of chylous ascites in adults.

Computed tomography of the abdomen is useful in identifying pathologic intra-abdominal lymph nodes and masses and is also helpful in determining the extent and localization of fluid, particularly if there is a suspicion of thoracic duct injury. Other diagnostic studies, such as lymphangiography and lymphoscintigraphy, can assist in detecting abnormal retroperitoneal nodes, leakage from dilated lymphatics, fistulization, and patency of the thoracic duct. Lymphangiography is the gold standard in defining cases of obstruction, although several complications have been described, including tissue necrosis, fat embolism, and hypersensitivity, all related to the volume and type of contrast used. In our patient, the primary lesion was found on an ultrasound. When she developed the chylous leakage, neither a CT scan nor lymphangiography contributed to either the diagnosis or the treatment.

Whilst having taken notion of the discussion in $2002{ }^{7}$ regarding the nomenclature of the "PEC" family of tumors, we still would like to refer to this tumor as a PEComa A subset of PEComas behave in a malignant fashion; a high mitotic index and the presence of necrosis, marked cytological atypia, and/or an infiltrative growth pattern appear to be associated with malignant behavior. Several of these features, including focal necrosis, a large size, and an elevated mitotic rate, were present in this case.

Because of the rarity of cases, effective treatment for malignant PEComa besides surgery is not yet known. Sporadic cases have been treated with chemotherapy with hardly any effect. ${ }^{8-10}$

In this patient, we had to deal with an additional clinical problem: in addition to the rare pathology, chyle was leaking until 2 months postoperatively. With our conservative treatment, this problem was adequately solved; however, the possible malignant fashion of the tumor has no further been explored.

Acknowledgments The authors thank Prof. C.D. Fletcher (Department of Pathology, Harvard Medical School, Boston) for reviewing the histology.

Open Access This article is distributed under the terms of the Creative Commons Attribution Noncommercial License which permits any noncommercial use, distribution, and reproduction in any medium, provided the original author(s) and source are credited.

\section{References}

1. Pan CC, Jong YJ, Chai CY, Huang SH, Chen YJ. Comparative genomic hybridization study of perivascular epithelioid cell tumor: molecular genetic evidence of perivascular epithelioid cell tumor as a distinctive neoplasm. Hum Pathol 2006;37(5):606-612.

2. Hornick JL, Fletcher CD. PEComa: what do we know so far? Histopathology 2006;48(1):75-82.

3. Folpe AL, Mentzel T, Lehr HA, Fisher C, Balzer BL, Weiss SW. Perivascular epithelioid cell neoplasms of soft tissue and gynecologic origin: a clinicopathologic study of 26 cases and review of the literature. Am J Surg Pathol 2005;29(12):1558-1575.

4. Dimmler A, Seitz G, Hohenberger W, Kirchner T, Faller G. Late pulmonary metastasis in uterine PEComa. J Clin Pathol 2003;56 (8):627-628.

5. Fukunaga M. Perivascular epithelioid cell tumor (PEComa) of soft tissue: case report with ultrastructural study. APMIS 2004;112 (2):98-104.

6. Almakdisi T, Massoud S, Makdisi G. Lymphomas and chylous ascites: review of the literature. Oncologist 2005;10(8):632-635.

7. Nuciforo PG, O'Hara CD. Correspondence re: Bonetti F, Martignoni G, Colato C, Manfrin E, Gambacorta M, Faleri M, et al. Abdominopelvic sarcoma of perivascular epithelioid cells. Report of four cases in young women, one with tuberous sclerosis. Mod Pathol 2001;14:563-568. And Tazelaar HD, Batts KP, Srigley JR. Primary extrapulmonary sugar tumor (PEST): a report of four cases. Mod Pathol 2001;14:615-622. Mod Pathol 2002;15(1):87-90.

8. Rigby H, Yu W, Schmidt MH, Fernandez CV. Lack of response of a metastatic renal perivascular epithelial cell tumor (PEComa) to successive courses of DTIC based-therapy and imatinib mesylate. Pediatr Blood Cancer 2005;45(2):202-206.

9. Chen IY, Yang SF, Chen FM, Chai CY. Abdominopelvic perivascular epithelioid cell tumor with overt malignancy: a case report. Kaohsiung J Med Sci 2005;21(6):277-281.

10. Jeon IS, Lee SM. Multimodal treatment using surgery, radiotherapy, and chemotherapy in a patient with a perivascular epithelioid cell tumor of the uterus. J Pediatr Hematol Oncol 2005;27 (12):681-684. 